Thorax (1969), 24, 695.

\title{
Basic physiopathological patterns of perfusion and inhalation pulmonary scintigraphy
}

\author{
M. FER RARI, A. PAEZ, C. LOPEZ SOTO, E. TOUYA, \\ R. BURGOS, AND J.J. Y TOUYA (H) \\ From the Nuclear Medicine Centre, School of Medicine, Montevideo, Uruguay
}

\begin{abstract}
${ }^{113 \mathrm{~m}}$ In is an excellent radiopharmaceutical for pulmonary scanning. Seven physiopathological patterns are described after the study of the results of successive perfusion and inhalation pulmonary scans-pulmonary artery obstruction, complete bronchial obstruction, partial bronchial obstruction, bronchiolar obstruction, anatomical changes in the pulmonary parenchyma, pathological pulmonary dead space, and intrapulmonary arterio-venous shunting. Pulmonary artery obstruction can be diagnosed early, precisely, and safely by this method. Pathological pulmonary dead space and intrapulmonary shunting have well-defined characteristics; the ability to demonstrate their topography and size in patients with pulmonary emphysema may lead to surgical removal of the affected regions, thus improving overall pulmonary function. The scintigraphic procedures used revealed that bronchial obstruction is associated with absent pulmonary arterial perfusion in the corresponding bronchial territory ; this finding is of the utmost importance, as it may permit the diagnosis of bronchial carcinoma before any radiological changes occur.
\end{abstract}

Pulmonary scintigraphy, which demonstrates the topographic distribution of perfusion and ventilation, is a useful method for evaluating regional lung function, and Ernst, Iglauer, Kronschwitz, and Spode (1958) were the first to use radioisotopes for this purpose. Taplin, Johnson, Dore, and Kaplan (1964) and Wagner, Sabiston, Iio, McAfee, Meyer, and Langan (1964) simultaneously developed the technique of pulmonary scintigraphy by perfusion of albumin macroaggregates labelled with ${ }^{131} \mathrm{I}$, or ${ }^{125} \mathrm{I}$, or ${ }^{51} \mathrm{Cr}$, or ${ }^{99 m}$ Tc. In 1966, Stern, Goodwin, Wagner, and Kramer introduced a technique of perfusion of particles of indium hydroxide $\left.{ }^{113 m} \mathrm{In}\right)$ plus iron hydroxide, these being easy to produce. Recently, Zolle, Rhodes, Buchanan, and Wagner (1967) published a new method of perfusion pulmonary scintigraphy, using albumin microspheres of uniform size and shape that could be labelled by adsorption with any radioisotope. When administered intravenously, these particles were retained, according to their diameter, in the pulmonary arterioles. The degree of accumulation in the different areas of the lung was in direct proportion to the regional blood flow, i.e., regions of high radioactivity were the better perfused.

In 1966, Taplin, Poe, and Greenberg described the technique of inhalation scintigraphy consisting of inhaling albumin radioaerosol labelled with
${ }^{99 m}$ Tc : this enabled them to study bronchial patency and the distribution of ventilation in the different regions of the lungs. The particles accumulated in the bronchioles and alveoli in direct proportion to the pulmonary ventilation, i.e., the regions of higher radioactivity were the better ventilated.

Therefore, with perfusion and inhalation pulmonary scintigraphy, the magnitude and extent of perfusion and ventilation of the pulmonary parenchyma could be evaluated.

The use of the two procedures in succession in normal individuals and in several pathological conditions has allowed us to describe some basic physio-pathological patterns by correlating pulmonary blood flow and ventilation in the different regions of the lung.

\section{MATERIALS AND METHODS}

Perfusion scintigraphy was performed with ${ }^{113 m}$ In prepared according to Stern et al. (1966). After resting for 10 minutes in the dorsal decubitus position, the patient was given an intravenous injection of $2 \mathrm{mCi}$. The scans were obtained with a Picker Magnascanner III. The speed of scan was $200 \mathrm{~cm} . / \mathrm{min}$. and the density of information was 800 counts/sq. cm.

The inhalation scintigraphy was performed with indium prepared by titrating the eluted $113 \mathrm{~m}$ In to pH 7.4 with $0.5 \mathrm{~N}$ sodium hydroxide. The aerosols 

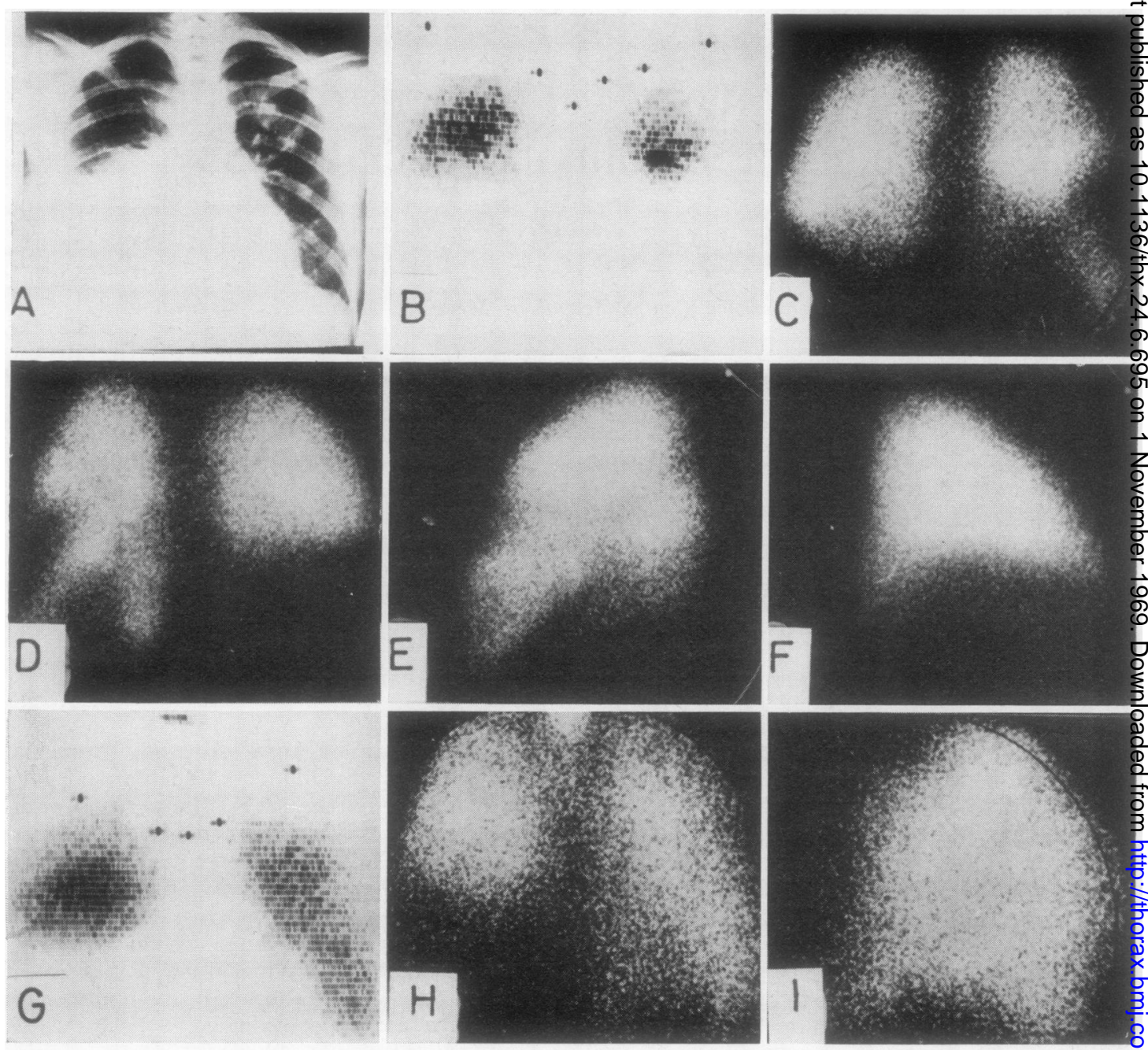

FIG. 1. Hepatic hydatid disease. Hydatid cysts and hydatid embolism in the left lung. (A) Chest film: right pleurad effusion; two hydatid cysts in the left upper lobe. (B) Perfusion scan, anterior view: wedge-shaped defect in the left lowert lobe. $(C, D, E)$ The same patient as seen with the Anger camera; anterior, dorsal, and left lateral views. $(F)$ Righ lateral view. (G) Inhalation scan. The aerosol penetrates freely throughout the lungs; the wedge-shaped defect is ne्य. seen. $(H, I)$ Anterior and left lateral views with the Anger camera.

were obtained with a Curry type micronebulizer connected to a Bird Mark 7 respirator. According to the manufacturers, the size of the particles thus obtained is 0.1 to 4 microns. The exhaled air was extracted by a hose to a fume hood.

Each patient inhaled $10 \mathrm{ml}$. of the solution (specific activity $2 \mathrm{mCi} / \mathrm{ml}$.). The patient was given an injection of $1 \mathrm{mg}$. of atropine subcutaneously 20 minutes before the inhalation to diminish bronchial and salivary secretions. After the inhalation the oropharynx was thoroughly rinsed with water and a glass of milk was drunk to remove any swallowed aerosol from the oesophagus. The scans were obtained with thæ⿸\zh14 same technique as for perfusion, but with a density of information of 400 counts $/ \mathrm{sq} . \mathrm{cm}$.

Rapid-sequence recording was made with an Angę camera $^{1}$ during inhalation, to study the penetratio and deposition of the aerosol in the tracheo-bronchial tract in normal individuals and in some pathologicap conditions.

Elimination of the radioaerosol was followed b three procedures: (a) performing rapid sequentiai

${ }^{1}$ Pho Gamma II. Nuclear, Chicago 
recordings of the lungs after the inhalation was completed; (b) measuring the decay of radioactivity in a projection area of pulmonary parenchyma over two hours; and $(c)$ measuring the radioactivity in blood samples and over the liver, to detect any early passage of ${ }^{113 m}$ In into the circulation.

\section{RESULTS}

Perfusion and inhalation scans in normal individuals showed a similar distribution of radioactivity. In both types there was more radioactivity in the lower third of both lungs than in the upper third. The hilar regions in the anterior, dorsal, and lateral views were hyporadioactive in the perfusion scan, while in the inhalation scan they were hyper-radioactive in comparison to the peripheral parenchyma.

In the inhalation scans $10-12 \%$ of the inhaled aerosol was detained in the pulmonary parenchyma; the rest was exhaled, deposited in the oropharynx, and sometimes swallowed. The serial scintigrams revealed that distribution of the aerosol was diffuse and homogeneous in both pulmonary fields from the start of inhalation; no bronchial deposits were observed.

The radioactivity curves, as measured by an Auger camera with a detector placed in the projection of the lower third of the right lung, showed a rise for the first 10 minutes and then a plateau. During a second inhalation another ascent was observed followed by another plateau 10 minutes later. Once inhalation was finished, the curve of pulmonary radioactivity declined with a semiperiod that was equal to the physical half-life of indium.

Blood samples obtained during the two hours following the inhalation showed no radioactive material in the systemic circulation; nor were there any changes in the hepatic background.

No difference was observed between the scans obtained with normal and with positive pressure inhalation in the normal individuals.

The following scintigraphic patterns can be described after study of the pathological cases:
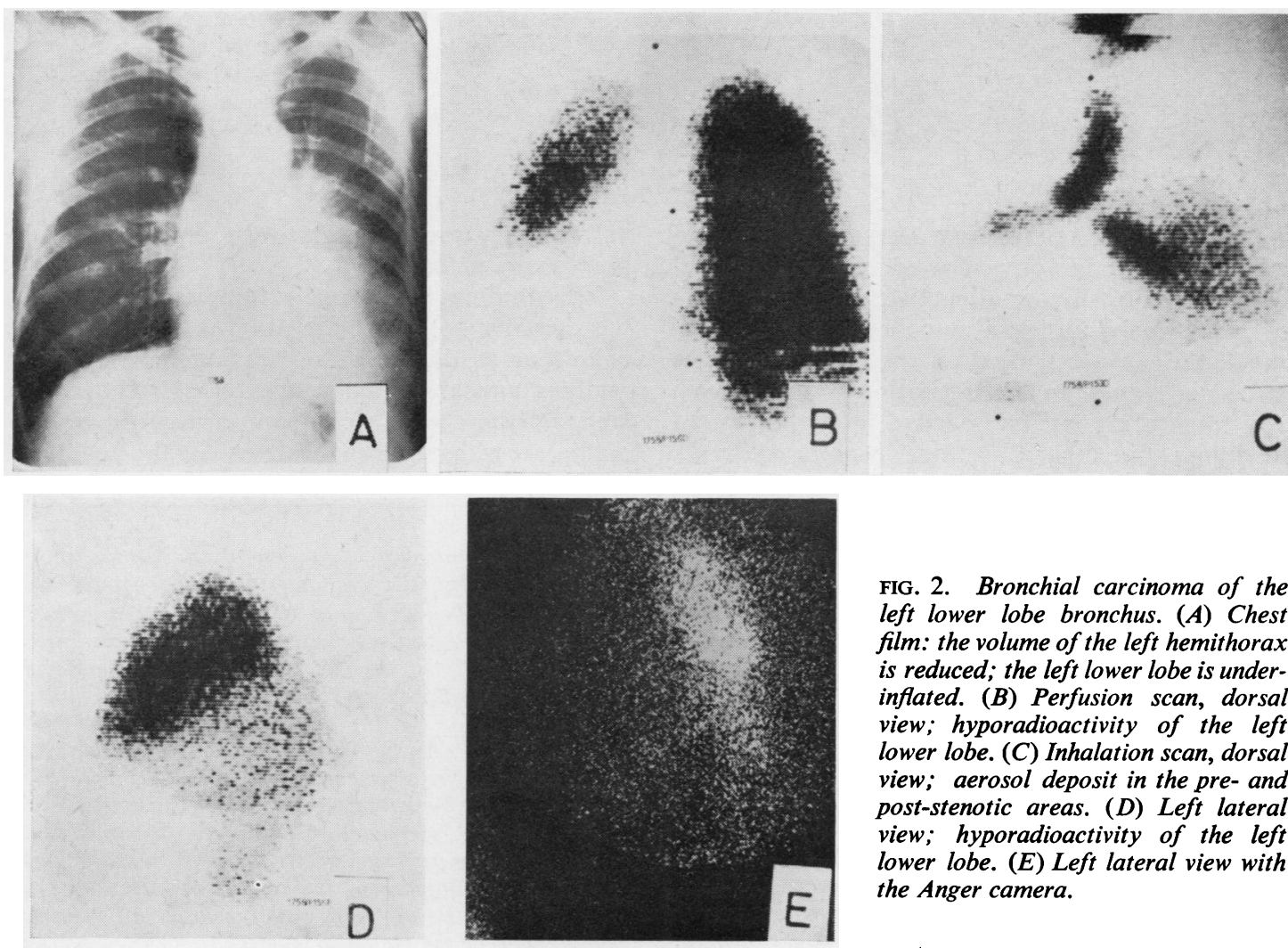

FIG. 2. Bronchial carcinoma of the left lower lobe bronchus. (A) Chest film: the volume of the left hemithorax is reduced; the left lower lobe is underinflated. (B) Perfusion scan, dorsal view; hyporadioactivity of the left lower lobe. $(C)$ Inhalation scan, dorsal view; aerosol deposit in the pre-and post-stenotic areas. (D) Left lateral view; hyporadioactivity of the left lower lobe. (E) Left lateral view with the Anger camera. 
OBSTRUCTION OF PULMONARY ARTERY Perfusion pulmonary scintigraphy showed either a wedgeshaped zone of hyporadioactivity with its apex towards the hilum or a punched-out peripheral defect, the size depending on the diameter of the obstructed vessel. In the inhalation scan any such area was barely less radioactive than the rest of the lung; the inhaled isotope penetrated freely into the zone of distribution of the obstructed artery, proving that ventilation had been maintained. The typical findings in obstruction of the pulmonary artery, such as that caused by embolism, were wedge-shaped or peripheral punchedout zones of hyporadioactivity in the perfusion scan that became radioactive after inhalation of the isotope (Fig. 1).

COMPLETE BRONCHIAL OBSTRUCTION Both perfusion and inhalation scans revealed absence of radioactivity in the zone corresponding to the obstructed bronchus, meaning that this sector was not perfused by the pulmonary artery. These studies have demonstrated that whenever a bronchus was obstructed, whatever its diameter,
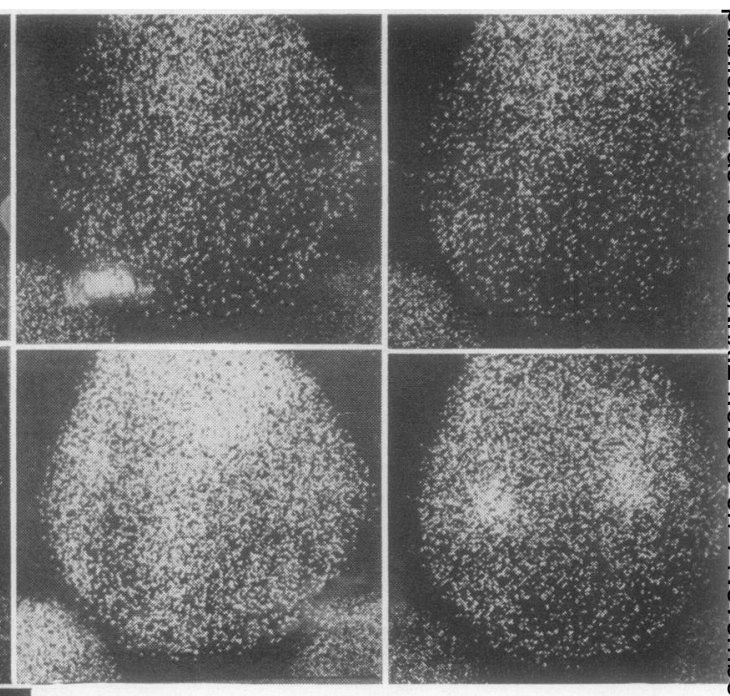

FIG. 3. Asthmatic attack. Serial scintigrams bronchiolar obstruction. Penetration of the radio aerosol and massive deposition in the main bronch and in the origins of the lobar bronchi.

its tributary area ceased to be perfused by the pulmonary artery.

This finding is extremely important because has demonstrated that perfusion scintigraphy maw enable us to diagnose lung cancer before abnor malities appear in the conventional radiographi 8 study of the chest: well-demonstrated hyporadio $\beta$ active areas have been observed in the region of

FIG. 4. Carcinoma of a division of the left upper lobse bronchus. $(A, B)$ Chest film, anterior and left lateral views: a slightly opacified high juxta-hilar lesion is seen in the le lung. $(C, D)$ Perfusion scan, anterior and dorsal view hyporadioactivity of the left lung. (E) Left lateral views note the hyporadioactive area in the anterior region of the left upper lobe. $(F, G, H)$ Inhalation scan, anterior, dorsas and left lateral views: there is a good distribution of the aerosol except for an angular hyporadioactive area in the anterior part of the left upper lobe. This case illustrates two scan patterns: segmental bronchial obstruction of the upper lobe (poor perfusion and ventilation) and pathologica pulmonary dead space of the rest of the left lung (poof perfusion with normal ventilation) caused by metastatio compression of the pulmonary artery. 
Basic physiopathological patterns of perfusion and inhalation pulmonary scintigraphy
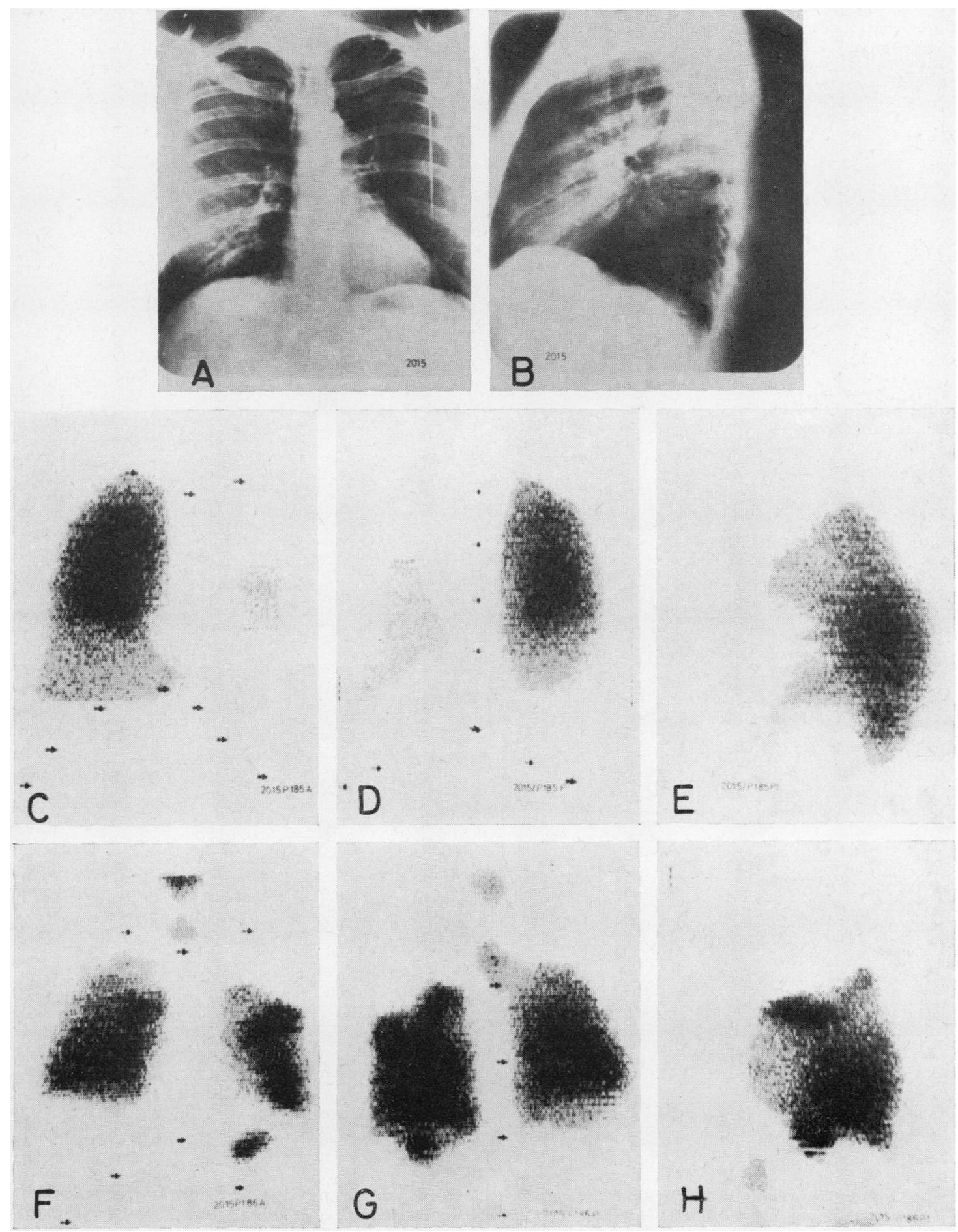

FIG. 4. Legend is at the foot of facing page. 


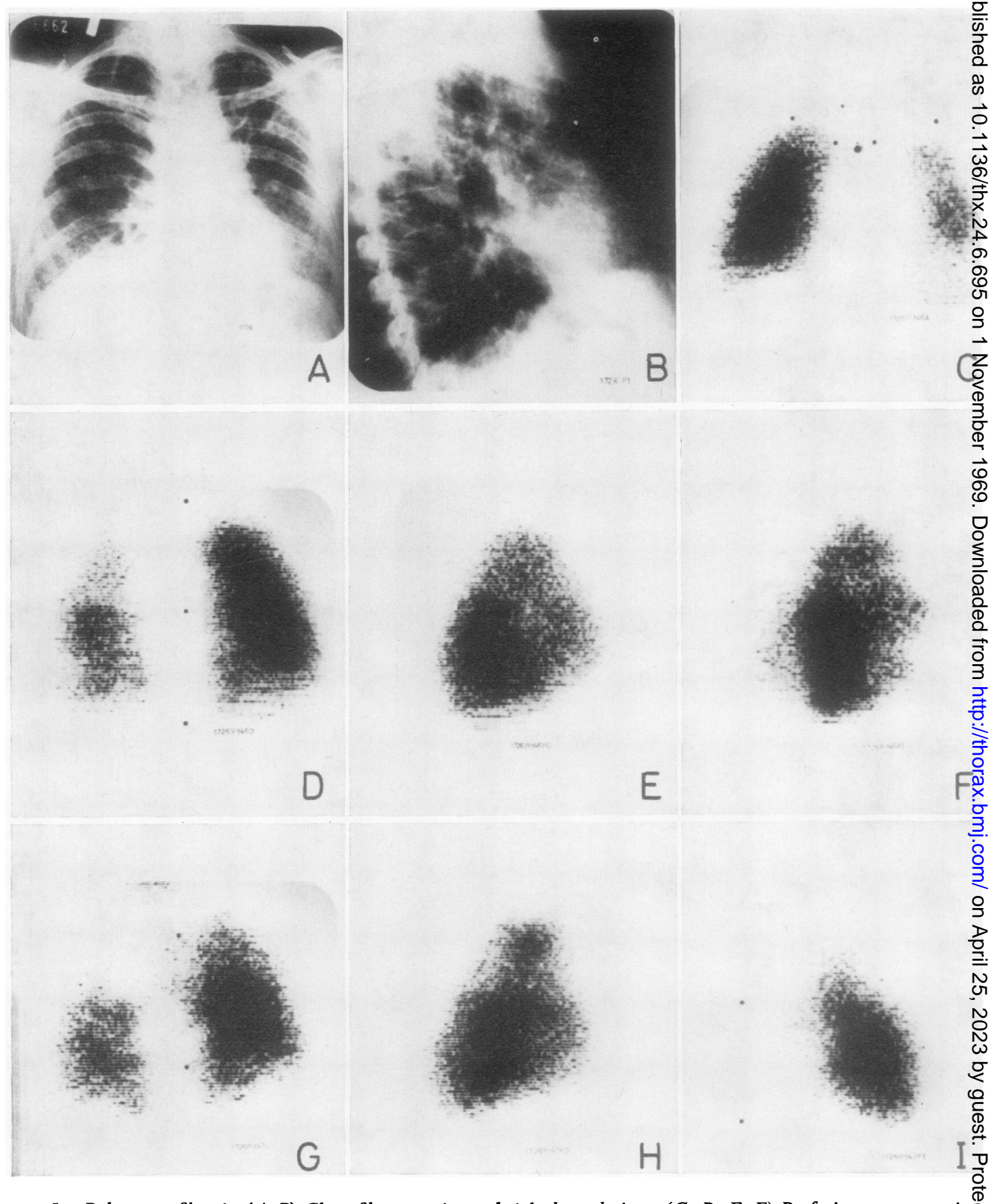

FIG. 5. Pulmonary fibrosis. $(A, B)$ Chest film, anterior and right lateral views. $(C, D, E, F)$ Perfusion scan, anterio dorsal, right lateral, and left lateral views. $(G, H, I)$ Inhalation scan, dorsal, right lateral, and left lateral views: not the hyporadioactivity of the upper lobes on the inhalation scans as compared with relatively good perfusion. This characteristic of intrapulmonary shunting. 
lung corresponding to the bronchus obstructed by the tumour (Feine, 1968 ; Maxfield, Hatch, and Ochsner, 1966).

PARTIAL BRONCHIAL OBSTRUCTION Hyporadioactivity in the sector corresponding to the partially obstructed bronchus was observed with both scintigraphic methods. The inhalation scan showed a pre- and post-stenotic radioaerosol deposit, this being the most significant scintigraphic finding in partial bronchial obstruction. It was caused by turbulence of the air flow adjacent to the obstruction (Taplin et al., 1964). In the serial scintigrams obtained with the Anger camera, the pre-stenotic deposit became visible after four minutes' inhalation (Fig. 2).

BRONCHIOLAR OBSTRUCTION This has been studied during asthmatic attacks. Perfusion scintigraphy shows peripheral hyporadioactive areas of variable size in both lungs. These were seen to change in both intensity and shape when serial studies were made, disappearing when the attack ceased. They were caused by decreased regional pulmonary arterial blood flow provoked by the bronchiolar obstruction (Mishkin, Wagner, and Tow, 1968 ; Taplin, Poe, Dore, and Greenberg, 1967).

Inhalation scintigraphy revealed a characteristic accumulation of the radioaerosol in the hilar and adjacent regions of both lungs. This was due to bronchiolar narrowing obstructing the passage of the aerosol to the alveoli (Fig. 3). If inhalation scintigraphy was performed after the attack had ceased the distribution of the aerosol was normal.

ANATOMICAL CHANGES IN THE PULMONARY PARENCHYMA Whenever disease altered normal pulmonary tissue, whatever its nature (inflammation, oedema, tumour, etc.) or mechanism (collapse, consolidation, rarefaction or destruction), both perfusion and inhalation scintigrams showed hyporadioactive areas with similar topography. This was the scintigraphic pattern most frequently observed, occurring in pneumonia, pleurisy, tumours, tuberculosis, emphysema, and hydatid cysts. It was similar to that caused by complete bronchial obstruction, although in the latter the topography was strictly lobar or segmental.

PATHOLOGICAL PUlmonary DEAD SPACE In obstructive emphysema the scan was generally of the type described in the previous paragraph. Perfusion and inhalation scintigraphy revealed hyporadioactive areas of differing sizes, irregularly distributed in both lungs but with a predominance in the upper lobes. In some cases, hyporadioactive areas of irregular and imprecise contours in the perfusion scan showed more radioactivity in the inhalation scan. These were therefore areas that were poorly perfused but well ventilated, thus demonstrating another physiopathological pattern of pulmonary scintigraphy-pathological pulmonary dead space (Fig. 4).

ARTERIO-VENOUS SHUNTS In pulmonary fibrosis, perfusion scans showed that there was a fairly uniform distribution of radioactivity throughout the lungs. Inhalation scintigraphy, on the contrary, revealed hyporadioactive areas of varying shape, size, and intensity, according to the different cases.

Therefore, there were areas that had good perfusion but insufficient ventilation; the blood that circulated through these hypoventilated areas acted as a shunt, causing arterial hypoxaemia. This is what occurs in pulmonary fibrosis and it explains the cyanosis in these patients, which was at one time attributed to aveolo-capillary block (Fig. 5).

\section{DISCUSSION}

The combination of perfusion and inhalation pulmonary scintigraphy used in succession is a valuable procedure for the diagnosis of physiopathological changes in the lung, as it evaluates semi-quantitatively regional lung perfusion and ventilation. ${ }^{113} \mathrm{~m}$ In hydroxide is an excellent radiopharmaceutical for the production of a radioaerosol for inhalation pulmonary scintigraphy. Its speed and ease of preparation, in comparison with albumin ${ }^{99 \mathrm{~m}} \mathrm{Tc}$, makes it the radioisotope of choice for this method.

Perfusion pulmonary scintigraphy is a procedure complementing conventional radiographic study of the chest in the physiopathological interpretation of different pulmonary conditions (Lopez-Majano and Wagner, 1968). However, it does not enable us to distinguish between such situations as regional ischaemia secondary to bronchospasm or to arterial obstruction from the permanent lesions of bullous emphysema.

Even if inhalation pulmonary scintigraphy does not measure pulmonary ventilation quantitatively, as does the xenon $\left({ }^{33} \mathrm{Xe}\right.$ ) technique (Ball, Stewart, Newsham, and Bates, 1962), it gives a useful idea of the regional distribution of ventilation and gives topographic and dynamic information about bronchial obstruction.

Using perfusion and inhalation pulmonary scintigraphy together, as described in this paper, improves the accuracy of either technique alone 
in investigating the physiopathological basis of every-day clinical problems. Thus, the following pulmonary scintigraphic patterns can be described :

(a) Anatomical or functional obstruction of the pulmonary artery: wedge-shaped or peripheral punched-out hyporadioactive areas in perfusion scintigraphy that are radioactive in the inhalation scan.

(b) Total bronchial obstruction: absence of radioactivity in the tributary area of the obstructed bronchus in both scans. According to the site of the obstruction, the topography of the hyporadioactive area will be pulmonary, lobar, or segmental.

(c) Partial bronchial obstruction: hyporadioactivity in the tributary area in both scans with deposits of radioaerosol in the pre- and poststenotic sites.

(d) Bronchiolar obstruction (bronchial asthma): multiple hyporadioactive areas with similar topography and intensity in both scans, that are transitory and change from study to study.

(e) Anatomical changes in the pulmonary parenchyma (collapse, consolidation, rarefaction, and destruction): absence of radioactivity in both scans in the involved area.

(f) Pathological pulmonary dead space: hyporadioactive areas in the perfusion scan that become normally radioactive after inhaling the isotope.

(g) Intrapulmonary shunt: areas showing greater radioactivity in the perfusion scan than in the inhalation scan. This regional difference of radioactivity is the consequence of a diminished ventilation/perfusion ratio.

These seven physiopathological scintigraphic patterns can exist individually, as in pulmonary embolism, bronchial stenosis, and pulmonary fibrosis, or be combined, as in chronic respiratory diseases with obstructed bronchi. In the latter condition the combination of patterns of fibrosis, bronchial obstruction, pathological pulmonary dead space, and intrapulmonary shunting is com-든 monly observed.

These findings have important therapeutic con $\frac{\overline{\bar{m}}}{-\frac{5}{8}}$ sequences. Pulmonary regions with shunts oro pathological dead space, that may disturb respiratory function and have an unfavourable effect onthe outcome of the illness, can be accurately located. The enlargement of the pulmonary vascu- $\vec{\omega}$ lar bed following the removal of bullae in emphy sema has been demonstrated by pre- and post $-\vec{x}$ operative perfusion scintigraphy (Lopez-Majanoj Tow, and Wagner, 1966).

\section{REFERENCES}

Ball, W. C., Jr., Stewart, P. B., Newsham, L. G. S., and Bates, D. v (1962). Regional pulmonary function studied with xenon 133. J. clin. Invest., 41, 519 .
(1962).

Ernst, H., Iglauer, E., Kronschwitz, H., and Spode, E. (1958) 으 Tierexperimentelle Untersuchungen zur Frage der Therapie von Lungentumoren mit Hilfe radioaktiver Gold-Kohle-Suspen-? sionen. Strahlenterapie, 107, 382.

Feine, U. (1968). Perfusion scintigraphy of the lungs. Germ. med. $M$ th. (English Language Edition), 13, 549 .

Lopez-Majano, V., Tow, D. E., and Wagner, H. N., Jr. (1966) Regional distribution of pulmonary arterial blood flow ino emphysema. J. Amer. med. Ass., 197, 81.

— and Wagner, H. N., Jr. (1968). Clinical application of lung scanning. Dis. Chest, 54, 356.

Maxfield, W. S., Hatch, H. B., and Ochsner, J. L. (1966). Perfusion lung scanning in evaluation of patients with bronchogenic carcinoma. Surg. Clin. N. Amer., 46, 1389.

Mishkin, F. S., Wagner, H. N., Jr., and Tow, D. E. (1968). Regional distribution of pulmonary arterial blood flow in acute asthma. J. Amer. med. Ass., 203, 1019.

Stern, H. S., Goodwin, D. A., Wagner, H. N., Jr., and Kramer, H. H. (1966). In ${ }^{113 m}$ - a short-lived isotope for lung scanning.O Nucleonics, 24, no. 10 (Oct.), p. 57.

Taplin, G. V., Johnson, D. E., Dore, E. K., and Kaplan, H. S. (1964). Organ visualization by photoscanning using micro and macro-E agregates of radioalbumin. In Medical Radioisotope Scanning. aggregates of radioald 20-24 April 1964 Vol. II, pp. 3-41. International Atomic Energy Agency, Vienna.

- Poe, H. D., and Greenberg, A. (1966). Lung scanning following radioaerosol inhalation. $J$. nucl. Med., 7, 77 .

- Dore, E. K., and Greenberg, A. (1967). Bronchial patency and aerated space assessment by scintiscanning. Lahey clin. 3 Found. Bull., 16, 297.

Wagner, H. N., Sabiston, D. C., Iio, M., McAfee, J. G., Meyer, J. K., and Langan, J. K. (1964). Regional pulmonary blood flow in man by radioisotope scanning. J. Amer. med. Ass., 187, 601.

Zolle, I., Rhodes, B. A., Buchanan, J. W., and Wagner, H. N., Jr.응 (1967). Properties and uses of radioactive albumin microspheres. J. nucl. Med., 9, 363. 